\title{
Atypisches Karpaltunnelsyndrom: Sonografie zum Nachweis einer isolierten Kompression des trifiden N. medianus
}

\section{Hintergrund \\ $\nabla$}

Die Nervensonografie mit hochfrequenten, hochauflösenden, linearen Sonden gilt als eine sensitive Methode zur Diagnosebestätigung eines Karpaltunnelsyndroms (KTS). Typisches sonografisches Charakteristikum einer Kompressionsneuropathie ist ein Kalibersprung des betroffenen Nerven an der Stelle der Kompression mit einer dadurch bedingten Verminderung der Nervenquerschnittsfläche (cross sectional area, CSA) bei transversaler Beschallung, und des Kalibers des betroffenen Nerven bei longitudinaler Beschallung. Typisch ist zudem eine proximal und seltener auch distal von der Kompression entstehende Zunahme des Nervendurchmessers. Die American Association of Neuromuscular and Electrodiagnostic Medicine (AANEM) bewertete in einem systemischen Review mit 45 Artikeln die Bedeutung der Sonografie für die Diagnose des KTS mithilfe der CSA. Aufgrund der Studien mit Klasse-I- und Klasse-II-Evidenz gilt demnach für die diagnostische Wertigkeit der Sonografie der Empfehlungsrad A beim KTS [1]. Obwohl die Sensitivität und Spezifität der Sonografie mit den weit verbreiteten elektrophysiologischen Untersuchungsverfahren vergleichbar sind, bleibt die Elektrophysiologie auch wegen des Ausschlusses anderer peripherer Nervenerkrankungen unverzichtbar. Sie dient der Quantifizierung der Nervenschädigung und der Verlaufsuntersuchungen unter Therapie.

Die Sonografie liefert einen zusätzlichen Beitrag zum Nachweis sekundärer Ursachen einer Nervenkompression und möglicher anatomischer Varianten, die operationsrelevant sein können. Bei fast jedem dritten symptomatischen Patienten wurde eine der folgenden anatomischen Varianten nachgewiesen [2]: hohe Teilung des N. medianus, A. mediana, atypisch verlaufender Thenarast, oder Lage der A. ulnaris sehr nah am N. medianus. Die anatomischen Varianten des N. medianus am Karpaltunnel wurden von Lanz in 4 Gruppen unterteilt [3]. Davon beinhaltet Gruppe 3 die hohe Teilung des N. medianus (sog. bifider N. medianus) , bei der der N. medianus sich schon am Unterarm oder im proximalen Karpaltunnel in 2 Stränge aufteilt und häufig von einer persistierenden A. mediana begleitet wird. Die klinische Bedeutung dieser bifiden Variante liegt darin, dass durch die isolierte Kompression meist des ulnar liegenden, kleinkalibrigeren Stranges eine atypische Symptomatik mit Symptomen in den Fingern III und IV vorliegen kann [4]. Die folgende Kasuistik beschreibt eine noch seltenere Variante, die isolierte Kompression des radial gelegenen Stranges eines trifiden N. medianus, die sensible Symptome des Fingers I distal des Versorgungsgebietes des Ramus palmaris $\mathrm{N}$. mediani hervorrief.

\section{Fallbericht $\nabla$}

Die 36-jährige Patientin klagte seit 12 Monaten über nächtliches Einschlafgefühl der re. Hand, seit 3 Monaten auch tagsüber. Zusätzlich gab sie Schmerzen am radialen Handgelenk an, besonders wenn sie mit der Computermaus arbeitete und berichtete über eine kontinuierliche Gefühlsstörung des Daumens. In der klinischen Untersuchung fand sich eine Hypästhesie der Volarseite des Daumenendglieds ( $\bullet$ Abb.1) und das Tinel-Zeichen war in Höhe der Handgelenksfalte am Thenar positiv. Darüber hinaus fanden sich keine sensiblen oder motorischen Ausfälle im Versorgungsgebiet des N. medianus.

In der Neurografie des $\mathrm{N}$. medianus rechts war die distale Latenzzeit (DLZ) mit 2,9 ms normal, und die sensible Nervenleitgeschwindigkeit (NLG) des N. medianus zwischen Handgelenk und Zeigefinger rechts lag mit $51 \mathrm{~m} / \mathrm{s}$ ebenfalls im Normbereich. Die sensible NLG des N. medianus zwischen Handgelenk und Daumen (bei dieser Ableitung lag die differente Elektrode am Daumengrundgelenk und die Reizung erfolgte am Handgelenk) war rechts jedoch mit $40 \mathrm{~m} / \mathrm{s}$ verlangsamt (zum Seitenvergleich: links betrug diese NLG $53 \mathrm{~m} / \mathrm{s}$ ), SNAP rechts $59 \mu \mathrm{V}$, links 62 $\mu \mathrm{V}$.

Die Nervensonografie zeigte am proximalen Karpaltunneleingang 3 distinkte Stränge des N. medianus. Nur der radial liegende Strang zeigte in Höhe der Handgelenksfalte am Eintritt in das Retinakulum eine segmentale Verdickung im transversalen und longitudinalen Schnitt-

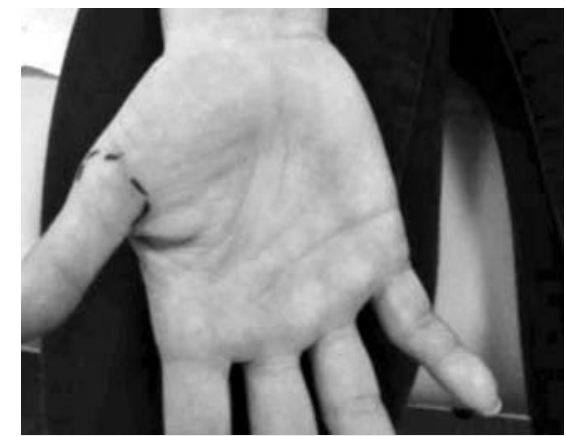

Abb. 1 Volare Hypästhesie der Daumenphalangen distal der eingezeichneten Punkte.

bild ( $\bullet$ Abb.2). Weiter proximal fand sich an der Stelle der Kompression eine hyperechogene Verdickung des umgebenden Gewebes als wahrscheinliche Kompressionsursache ( Abb.3). Die beiden weiter medial verlaufenden Äste waren morphologisch unauffällig ( $\bullet$ Abb.4). Auch weiter distal im Karpaltunnel fanden sich keine Kompressionszeichen.

\section{Fazit}

$\nabla$

Die B-Bildsonografie ist eine schnell durchzuführende und kostengünstige bildgebende Methode zur Untersuchung der anatomischen Varianten des N. medianus. Das ist klinisch vor allem für die Diagnostik eines vermuteten Karpaltunnelsyndroms (KTS) hilfreich. Ein trifider N. medianus wurde sonografisch schon mehrmals als anatomische Variante asymptomatischer Patienten beschrieben, ebenso ein trifider N. medianus eines $\mathrm{Pa}$ tienten mit bilateralem KTS, oder mit der Variante einer persistierenden A. mediana [5-7], nicht jedoch die isolierte Kompression eines seiner Stränge. Der Ramus palmaris N. mediani, der den Daumenballen sensibel innerviert, kann sonografisch ebenfalls dargestellt werden. Dieser verlässt den Hauptstamm des N. medianus jedoch in verschiedener Höhe schon im distalen Unterarmdrittel und läuft nicht durch das Retinaculum flexorum, kann also nicht durch einen verengten Karpaltunnel geschädigt werden. Die sonografische Lokalisationsdiagnostik erlaubt in dem hier dargestellten Fall nicht nur eine gezielte chirurgische Behandlung der Kompressionsstelle, sondern auch eine 


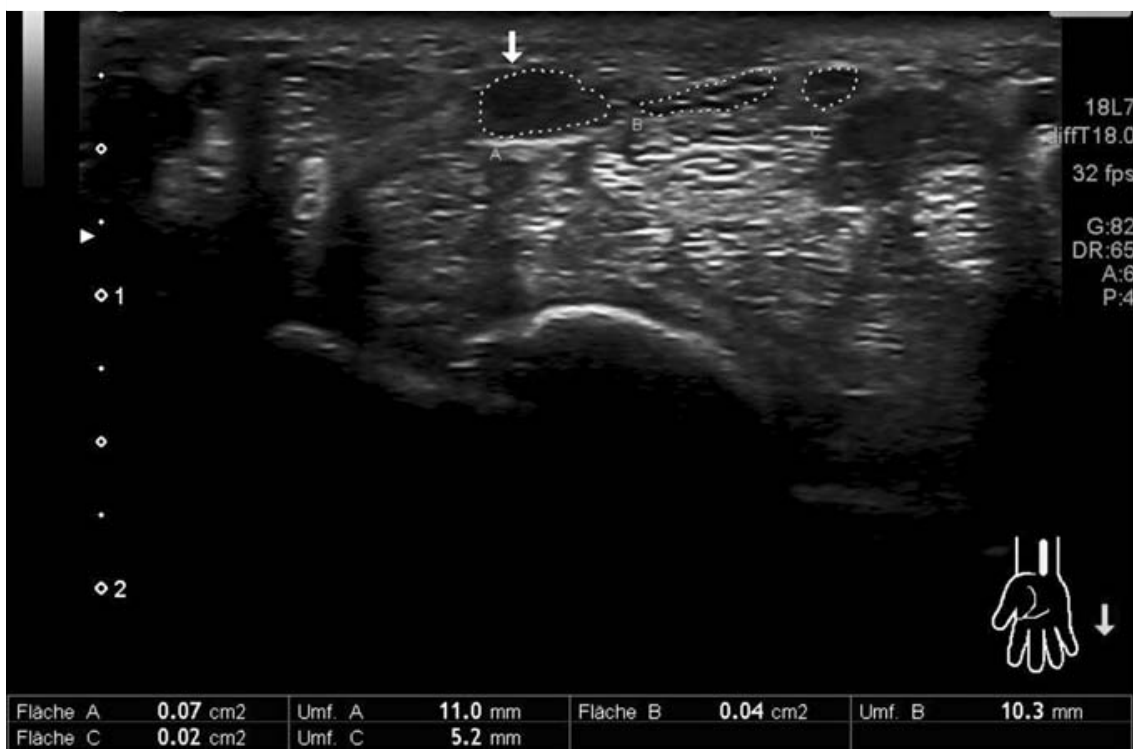

Abb.2 Isolierte Erweiterung des radial gelegenen Stranges (Pfeil) des trifiden N. medianus (CSA 0,07 $\mathrm{cm}^{2}$ ) bei transversaler Beschallung in Höhe des Karpaltunneleingangs. Die beiden weiter ulnar liegenden Nervenstränge sind normal groß.

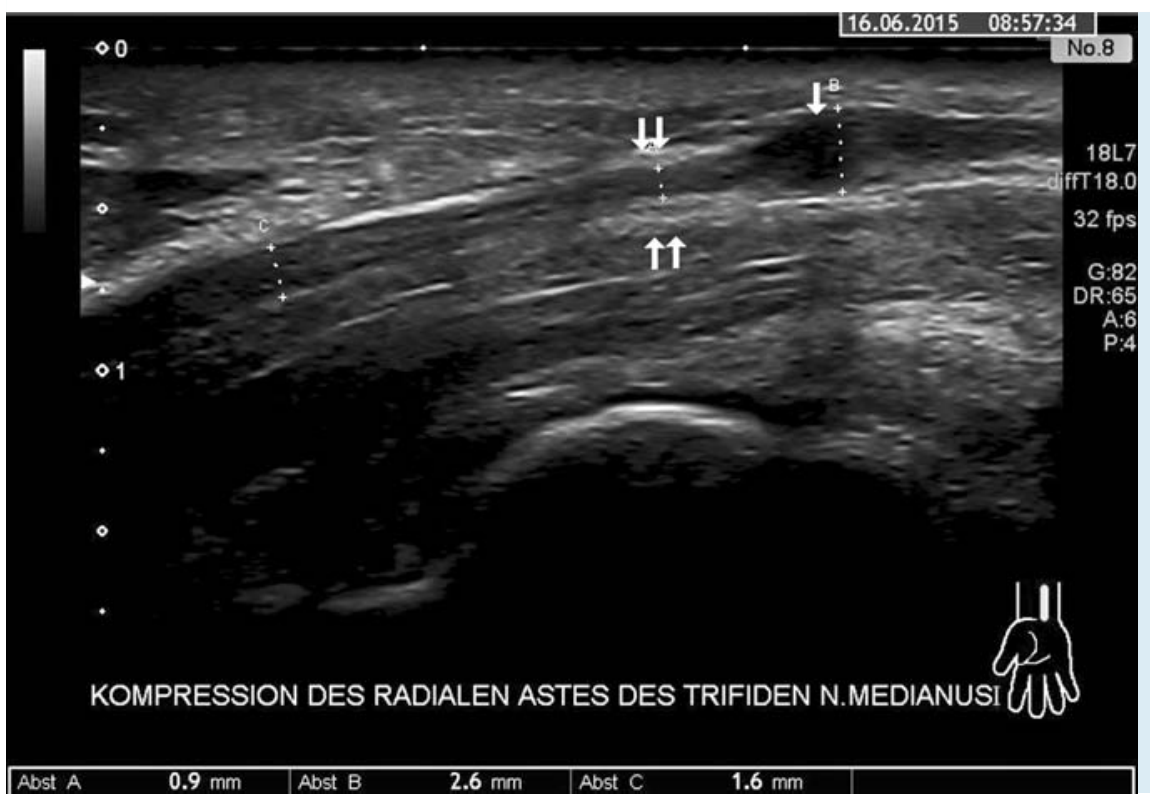

Abb.3 Ultraschallbild des radial liegenden Stranges des trifiden N. medianus im Längsschnitt. Segmentale Kaliberänderung von $0,9 \mathrm{~mm}$ (Messpunkt A) auf 2, $6 \mathrm{~mm}$ (Messpunkt B). Das Pfeil zeigt die proximal der Kompression liegende Erweiterung des Nervenastes. Die Doppelpfeile zeigen auf die hyperechogene Verdickung des umliegenden Gewebes an der Stelle der Kompression.

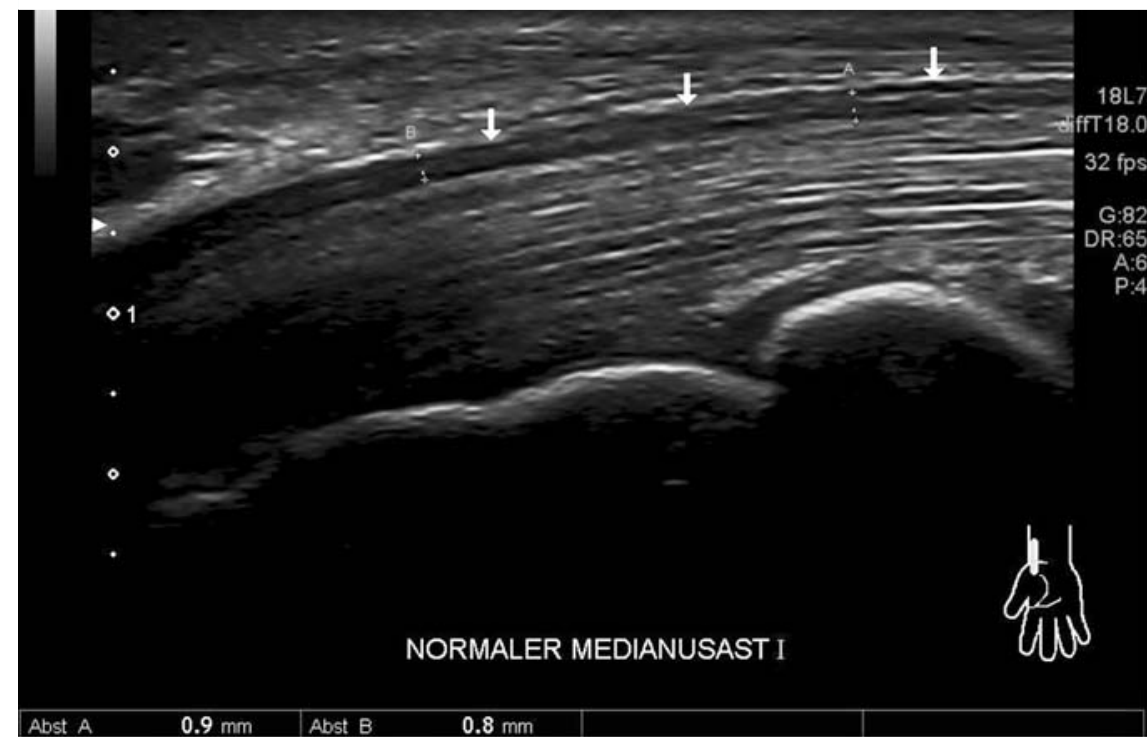

Abb.4 Ultraschallbild des mittleren Stranges des trifiden N. medianus (Pfeile) im Längsschnitt. Kein Nachweis einer segmentalen Kompression oder Auftreibung. 
Vermeidung der iatrogenen Verletzung der übrigen Endäste.

\section{Interessenkonflikt}

Der Autor gibt an, dass kein Interessenkonflikt besteht.

\section{Literatur}

1 Cartwright M, Hobson-Webb L, Boon A et al. Evidence-based guideline: Neuromuscular ultrasound for the diagnosis of carpal tunnel syndrome. Muscle Nerve 2012; 46: 287 293

2 Kele $H$, Verheggen R, Bittermann $H J$ et al. The potential value of ultrasonography in the

\section{Möchten Sie von einem besonderen Bild aus Ihrer Klinik berichten?}

Autorenhinweise finden Sie unter:

http://www.thieme.de/de/klinische-neurophysiologie/

autoren-1869.htm

Schicken Sie Ihre Beiträge an: klinneuro@thieme.de. evaluation of carpal tunnel syndrome. Neurology 2003; 61: 389-391

3 Lanz U. Anatomical variations of the median nerve in the carpal tunnel. J Hand Surg 1977: $2: 44-53$

4 Reimers $C D$, Gaulrapp H, Kele H, Hrsg. Sonographie der Muskeln, Sehnen und Nerven. Köln.: Deutscher Ärzte-Verlag; 2004: 254

5 Yalcin E, Onder B, Akyuz M. Trifid median nerve. J Hand Surg Eur Vol 2011; 36: 812 813

6 Duymus M, Yilmaz O, Ulasli AM et al. Coexistence of trifid and bifid median nerve in a patient with bilateral carpal tunnel syndrome. Turk Neurosurg 2013; 23: 685-687

7 Chávez-López MA, Tello-Esparza A. Trifid median nerve associated with persistent median artery. J Clin Rheumatol 2015; 21: 102

\section{Bibliografie}

DOI http://dx.doi.org/

10.1055/s-0035-1552697

Online-Publikation: 30.7.2015

Klin Neurophysiol 2015; 46: 155-157

(c) Georg Thieme Verlag KG

Stuttgart · New York

ISSN 1434-0275 